\title{
Supporting Information-4
}

Simultaneous Construction of Silica Nanotubes Loaded with Pd Nanoparticles for Catalytic Hydrodechlorination of Chlorophenols

Tao Hu, ${ }^{\dagger}$ Tingting Liu, ${ }^{\dagger}$ Caiyun Xu, Mingxi Liu, Shenghu Zhou*

Shanghai Key Laboratory of Multiphase Materials Chemical Engineering, School of

Chemical Engineering, East China University of Science and Technology, 130

Meilong Road, Shanghai 200237, P. R. China.

$\dagger$ These authors are equal contributors

*Corresponding author:

E-mail: zhoushenghu@ecust.edu.cn

Fax: (+86) 21-64253159 for S. Z. 
The followings are. $\mathrm{dm} 3$ files of Figure $3 \mathrm{a}$ in the manuscript.

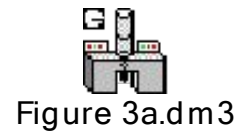

